INPLASY

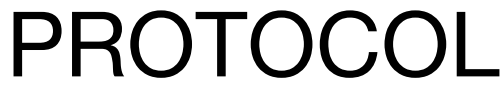

To cite: Zhou et al.

Acupuncture related therapy

for ovulatory disorders: a

systematic review and

Bayesian network meta-

analysis protocol. Inplasy

protocol 202160078. doi:

10.37766/inplasy2021.6.0078

Received: 22 June 2021

Published: 22 June 2021

Corresponding author:

Linwen Deng

dlwen1982@163.com

Author Affiliation:

Hospital of Chengdu

University of traditional

Chinese Medicine.

Support: National Natural

Science Found.

Review Stage at time of this submission: Preliminary

searches.

Conflicts of interest:

None declared.

\section{Acupuncture related therapy for ovulatory disorders: a systematic review and Bayesian network meta- analysis protocol}

Zhou, $\mathrm{H}^{1}$; Yan, L2; Zhang, SG3; Yang, Y4; Zheng, XY5; Wang, $\mathrm{H}^{6}$; Deng, $L^{7}$.

Review question / Objective: Ovulation disorder is the main cause of infertility, and its harm has aroused international attention. A large number of clinical studies have shown that acupuncture therapy has potential application value in ovulation disorders. This study aims to evaluate the effectiveness and safety of different acupuncture therapies through systematic review and Bayesian network metaanalysis (NMA).

Study designs to be included: Randomized controlled trials (RCTs) will be included.

Information sources: PubMed, EMBASE, Cochrane Library, CBM, CNKI, Wan Fang, and VIP were searched by computer. The search time was from the establishment to 2021.

INPLASY registration number: This protocol was registered with the International Platform of Registered Systematic Review and Meta-Analysis Protocols (INPLASY) on 22 June 2021 and was last updated on 22 June 2021 (registration number INPLASY202160078).

\section{INTRODUCTION}

Review question / Objective: Ovulation disorder is the main cause of infertility, and its harm has aroused international attention. A large number of clinical studies have shown that acupuncture therapy has potential application value in ovulation disorders. This study aims to evaluate the effectiveness and safety of different acupuncture therapies through systematic review and Bayesian network metaanalysis (NMA).

Condition being studied: Normal ovulation requires the normal function of the 
hypothalamus-pituitary ovary gonadal axis. Dysfunction of any link, or organic lesions, can cause temporary or long-term ovarian dysfunction, leading to abnormal ovulation. The causes of ovulation disorder include: (a)anovulation of central nervous system; (b)Hypothalamic anovulation; (c)Pituitary anovulation; (d)Ovarian anovulation; (e)Polycystic ovary syndrome $[8,9,10]$; (f) Luteinized unruptured follicle syndrome; (g)Others: other endocrine systems beyond gonadal axes, such as thyroid and adrenal cortex dysfunction, and some systemic diseases, such as severe malnutrition, can affect ovarian function and lead to ovulation dysfunction. Acupuncture is a traditional medical treatment in China. Studies have shown that acupuncture treatment of ovulatory disorders has a significant effect, the long-term effect is lasting, and the adverse reactions are small. However, other studies hold an objection that the effectiveness of acupuncture in the treatment of the disease remains to be proved, and the quality of evidence-based clinical observation is low. Due to the variety of acupuncture-related therapies, different emphasis on efficacy, inconsistent conclusions, and lack of direct comparison between different acupuncture-related therapies, it is not conducive to clinical promotion and the choice of the best treatment scheme. Therefore, in addition to the conventional meta-analysis method, this study further uses the network metaanalysis method to compare the efficacy of a variety of acupuncture related methods on ovulation rate and pregnancy rate of patients with ovulation disorder and sort the treatment measures according to the efficacy, to provide evidence-based medicine for a clinical selection of the best acupuncture related treatment scheme to improve the indicators of patients with ovulation disorder. Network meta-analysis (NMA) uses the technique of adjusting indirect comparison to make the direct and indirect cross-comparison. It can also compare the efficacy of at least two interventions by combining data from different trials, to rank all the included interventions relatively, Therefore, it is also called mixed treatment comparison meta- analysis or multiple treatments metaanalysis. The purpose of this paper is to compare the effects of various acupuncture related measures on ovulation rate and pregnancy rate of patients with ovulation disorders by using the network metaanalysis method, to provide a reference for clinical application.

\section{METHODS}

Participant or population: Patients with ovulation disorders refer to the disease types published by upto date (www.update.com) At the same time, combined with world health organization(WHO) standards, ovulation disorders are divided into four categories: (a) WHO I type ovulation disorder: the lesion is in hypothalamus or pituitary, which is characterized by low level of endogenous estrogen, low level of FSH and LH. (b) WHO II type: the performance of endogenous $\mathrm{FSH}$, LH levels imbalance, common in patients with polycystic ovary syndrome. (c) WHO III patients: ovarian failure, the performance of $\mathrm{FSH}$, LH levels increased, estrogen levels decreased. (d) Excluding the above three types of ovulation disorders, such as hyperprolactinemia, luteinized unruptured follicle syndrome and so on. Patients with severe medical conditions were excluded. Patients with ovulatory disorders.

Intervention: The treatment group was treated with all kinds of acupuncturerelated therapies, including electroacupuncture, auricular acupuncture, warm acupuncture, auricular point sticking and pressing, moxibustion, acupoint catgut embedding, etc; The frequency of acupoint use and the frequency of intervention measures were counted and displayed by histogram. We show the possible patterns of different intervention methods acupuncture points ovulation disorders, and show the statistics of the number of acupuncture points for different diseases and different acupuncture programs.The treatment group was treated with all kinds of acupuncture-related therapies, including electroacupuncture, auricular acupuncture, warm acupuncture, auricular point sticking 
and pressing, moxibustion, acupoint catgut embedding, etc; The control group was Western medicine or placebo, or the comparison of various acupuncture related therapies. The frequency of acupoint use and the frequency of intervention measures were counted and displayed by histogram. We show the possible patterns of different intervention methods acupuncture points ovulation disorders (2-A), and show the statistics of the number of acupuncture points for different diseases and different acupuncture programs.

Comparator: The control group was Western medicine or placebo, or the comparison of various acupuncture related therapies.

Study designs to be included: Randomized controlled trials (RCTs) will be included

Eligibility criteria: The following cases were considered not to meet the inclusion criteria:(a) Abstracts, conference papers, dissertations, Case reports, animal mechanism studies, etc; (b) Repetitive publications; (c) There were no definite diagnostic criteria and efficacy evaluation criteria; (d) There is no definite randomized method in literature; (e) The intervention measures did not meet the requirements. (f)Articles that were not available after contacting their authors. (i)Articles published for different indicators in the same study were merged.

Information sources: PubMed, EMBASE, Cochrane Library, CBM, CNKI, Wan Fang, and VIP were searched by computer. The search time was from the establishment to 2021.

Main outcome(s): The main outcome was ovulation rate or hormonal outcome. Different ovulation disorders will be discussed separately.

Additional outcome(s): The incidence of adverse events that are directly related to interventions that evaluate safety measures will be considered as secondary outcomes.
Quality assessment / Risk of bias analysis: Two researchers evaluated the included studies according to the bias risk assessment tool recommended in Cochrane Handbook 5.1. (1)Random sequence generation; (2)Allocation concealment; (3)Blinding of participants and personne; (4)Blinding of outcome assessment; (5)Incomplete outcome data; (6)Selective reporting; (7)other bias its altogether seven items; If the two sides do not agree, they will discuss with the third researcher to reach an agreement.

Strategy of data synthesis: Two researchers independently screened, extracted, and cross-checked the literature. In case of disagreement, both parties shall reach an agreement through discussion or the third researcher shall assist in the ruling. Data were extracted by a unified data extraction table, including title, author, sample size, average age, gender, course of the disease, intervention measures, course of treatment, outcome indicators, etc. The selection process will be performed in the PRISMA flowchart.

Subgroup analysis: If the relevant studies are sufficient, the following characteristics will be considered for subgroup analysis: (a) RCT studies of the same type of ovulation disorder but with different diseases or causes should be analyzed; (b) RCT studies of different acupuncture intervention methods for the same disease should be analyzed; (c) RCT studies of different acupuncture points for the same treatment method should be analyzed.

Sensitivity analysis: Sensitivity analysis was performed by sequentially deleting trials to check the stability of the primary outcomes.

Country(ies) involved: China.

Keywords: Acupuncture and moxibustion therapy; Ovulation disorders; Network meta-analysis (NMA).

Dissemination plans: To promote the application of acupuncture and moxibustion in international Gynecology, 
and to screen out and recommend the best treatment combination.

Contributions of each author:

Author 1 - Hang Zhou - The author drafted the manuscript.

Email: zh6463@foxmail.com

Author 2 - Li Yan.

Email: 309617316@qq.com

Author 3 - Shuguang Zhang.

Author 4 - Yi Yang.

Email: 1971589776@qq.com

Author 5 - Xiaoyan Zheng.

Author 6 - Huan Wang.

Author 7 - Linwen Deng - The author was responsible for the design of the whole study.

Email: dlwen1982@163.com 